Pacific Journal of Mathematics

AN APPLICATION OF THE BOTT SUSPENSION MAP TO THE 


\section{AN APPLICATION OF THE BOTT SUSPENSION MAP TO THE TOPOLOGY OF EIV}

\section{LAWRENCE CONLON}

Consider the compact simply connected symmetric pair $\left(E_{6}, F_{4}\right)$. By a slight abuse of the notation of $\mathbf{E}$. Cartan, the corresponding symmetric space is denoted by $E I V$. Let $W$ be the Cayley projective plane. The Bott suspension map $E: \Sigma(W) \rightarrow E I V$ (where $\Sigma$ denotes the nonreduced suspension) is defined by means of the set of minimal geodesic segments joining the two nontrivial points of the "center" of $E I V$. In this paper a map $q: S^{25} \rightarrow \Sigma(W)$ is constructed and $E$ is extended to a homeomorphism of $\Sigma(W) \cup{ }_{q} e_{26}$ onto $E I V$. Among other things, this gives canonical isomorphisms $\pi_{j}(E I V) \approx$ $\pi_{j}(\Sigma(W)), 0 \leqq j \leqq 24$. These groups are explicitly determined.

Statement of results. The maps $E$ and $q$ will be constructed in $\S 2$ and the following theorems will be proven.

THEOREM 1.1. The map $E$ extends to a homeomorphism $E^{\prime}$ : $\Sigma(W) \bigcup_{q} e_{26} \rightarrow E I V$.

CoROLlary 1.2. $E_{*}: \pi_{j}(\Sigma(W)) \rightarrow \pi_{j}(E I V)$ is a bijection for $j \leqq 24$, and a surjection for $j=25$.

TheOREM 1.3. $\operatorname{Im}\left(q_{*}\right)=\operatorname{Ker}\left(E_{*}\right)$ in homotopy in dimensions $\leqq$ 32 , and

$$
0 \longrightarrow \pi_{25}\left(S^{25}\right) \underset{q_{*}}{\longrightarrow} \pi_{25}(\Sigma(W)) \underset{E_{*}}{\longrightarrow} \pi_{25}(E I V) \longrightarrow 0
$$

is exact and canonically split, with $\pi_{25}(E I V)$ a finite 2-primary group.

Having by (1.2) reduced the problem of computing $\pi_{j}(E I V), j \leqq$ 24 , to a somewhat easier problem, we devote the remaining sections of the paper to deducing the consequences listed below. We do not list $\pi_{j}(E I V)$ for $j \leqq 15$, since isomorphisms $\pi_{j}(E I V) \approx \pi_{j}\left(S^{9}\right)$, together with the explicit values of these latter groups, are already well known for that range.

$$
\begin{aligned}
& \pi_{16}(E I V)=0 \\
& \pi_{17}(E I V)=\boldsymbol{Z}+\left(\boldsymbol{Z}_{2}\right)^{2} \\
& \pi_{18}(E I V)=\left(\boldsymbol{Z}_{2}\right)^{3}
\end{aligned}
$$




$$
\begin{aligned}
& \pi_{19}(E I V)=\boldsymbol{Z}_{6} \\
& \pi_{20}(E I V)=\boldsymbol{Z}_{1512}+\boldsymbol{Z}_{2} \\
& \pi_{21}(E I V)=0 \\
& \pi_{22}(E I V)=\boldsymbol{Z}_{3} \\
& \pi_{23}(E I V)=\boldsymbol{Z}_{4} \\
& \pi_{24}(E I V)=\boldsymbol{Z}_{225}+(2 \text {-primary group }) .
\end{aligned}
$$

REMARKS. (1.4) was communicated to the author some time ago by Shôrô Araki who proved it by a somewhat different method (unpublished). The present paper actually resulted from attempts to verify this formula. (1.9) was proven in a different way in [8] and (1.5) and (1.10) remove the ambiguities from the partial determinations of these groups in that same paper. In (1.1) one gets a fully explicit cellular structure by recalling that

$$
\Sigma(W)=e_{0} \mathbf{U}_{p} e_{9} \mathbf{U}_{g} e_{17}
$$

where $p: S^{8} \rightarrow e_{0}$ is the only map possible and $g: S^{16} \rightarrow e_{0} \bigcup_{p} e_{9} \approx S^{9}$ is the suspension of the standard Hopf map $f: S^{i 5} \rightarrow S^{8}$.

In the course of this paper we will repeatedly (and without further reference) make use of the values of $\pi_{i}\left(S^{n}\right)$ as found in [14].

2. The maps $E$ and $q$. Let $\mathfrak{e}_{6}$ be the Lie algebra of $E_{6}$ and $\beta: \mathfrak{e}_{6} \rightarrow \mathfrak{e}_{6}$ the involution corresponding to $E I V$. Let $\mathfrak{m} \subset \mathfrak{e}_{6}$ be the -1 eigenspace of $\beta$. Let $\mathrm{t} \subset \mathrm{m}$ be a maximal abelian subalgebra (a two dimensional real vector space) and consider the root system of $E I V$ relative to t. This is a proper root system (in the sense of [2]) isomorphic to the root system of $A_{2}$, each root having multiplicity 8 . Let $\Delta$ be a fundamental simplex in $\mathrm{t}$.

The symmetric space $E I V$ is canonically imbedded in $E_{6}$ as $\exp (\mathfrak{m})$. The adjoint action of $F_{4}$ on $\mathfrak{n}$ passes over, under exp, to the adjoint action of $F_{4}$ on $E I V \subset E_{6}$.

$\operatorname{Exp} \mid \Delta$ is one-to-one (since $E I V$ is simply connected) and exp (A) intersects each $F_{4}$-orbit on $E I V$ in one and only one point.

Let $B$ denote the union in $\mathrm{m}$ of the $F_{4}$-orbits of points of $\Delta$. By the above remarks exp: $B \rightarrow E I V$ is onto. Let $s(t), 0 \leqq t \leqq 1$, describe the edge of $\Delta$ opposite the vertex 0 . Then $x_{0}=\exp (s(0))$ and $x_{1}=$ $\exp (s(1))$ coincide with the nontrivial elements of the center $Z_{3}$ of $E_{6}$, while expos is a minimal geodesic joining $x_{0}$ and $x_{1}$. The following lemma and its corollary are completely straightforward. 
LEMMA 2.1. $B$ is homeomorphic to the standard closed cell $e_{26}$ and the boundary $\partial B \approx S^{25}$ is the union of the $F_{4}$-orbits of $s(t), 0 \leqq$ $t \leqq 1$.

Corollary 2.2. Under the homeomorphism $B \approx e_{26}$, exp $\mid B$ defines a surjection $e_{26} \rightarrow E I V$ which is a homeomorphism on the interior of $e_{25}$.

LEMMA 2.3. $\exp (\partial B) \approx \Sigma(W)$.

Proof. From [1] one knows that the centralizer in $F_{4}$ of $\exp (s(t))$, $0<t<1$, is the symmetric subgroup Spin (9) $\subset F_{4}$, while for $t=0,1$ the centralizer is clearly all of $F_{4}$. Since $W=F_{4} /$ Spin (9), the lemma follows.

COROllary 2.4. The inclusion $\exp (\partial B) \subset E I V$ is a Bott suspension $E: \Sigma(W) \rightarrow E I V$.

Proof. Let $\Omega=\Omega\left(E I V ; x_{0}, x_{1}\right)$, the space of paths on $E I V$ joining $x_{0}$ and $x_{1}$. From the proof of $(2.3)$ it is clear that the subspace of shortest geodesics in $\Omega$ is homeomorphic to $W$. The adjoint of the inclusion map $W \subset \Omega$ is precisely the Bott suspension [4], is one-to-one, and its image is $\exp (\partial B)$.

Of course, we define $q$ as $\exp \mid \partial B$ and immediately obtain (1.1) and (1.2).

REMARK. The loop space $\Omega$ of $E I V$ is homology commutative, hence the theory of [5] can be applied to the Pontrjagin ring $H_{*}(\Omega)$. $W \subset \Omega$ proves to be a generating variety contributing generators $x_{8}, x_{16} \in H_{*}(\Omega) \approx Z\left[x_{8}, x_{16}\right], \operatorname{dim}\left(x_{i}\right)=i$. The diagram

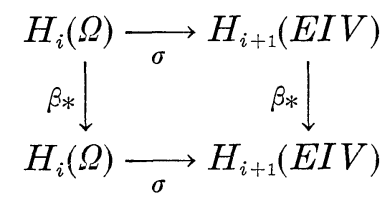

is commutative, where $\sigma$ is homology suspension and the homomorphisms $\beta_{*}$ are induced by the involution $\beta$ of $E_{6} . \quad \beta_{*}$ is -1 on $H_{9}(E I V) \approx Z$ [9] and $\sigma\left(x_{8}\right)$ generates this group. Thus $\beta_{*}\left(x_{8}\right)=-x_{8}$ and $\beta_{*}\left(x_{8}^{2}\right)=x_{8}^{2}$. $\beta_{*}$ is -1 on $H_{17}(E I V) \approx \boldsymbol{Z}$ [9], so $\sigma\left(x_{8}^{2}\right)=0$. $\sigma H_{16}(\Omega)=H_{17}(E I V)$, hence $\sigma\left(x_{16}\right)$ generates that group. From the known homology of $E I V$ [9], it follows that $E_{*}: H_{i}(\Sigma(W)) \rightarrow H_{i}(E I V)$ is bijective, $i \leqq 25$. (1.2) then follows by the Whitehead theorem. One can also deduce a map $q$ (defined 
up to homotopy) and a weakened version of (1.1) in which $E^{\prime}$ is only a homotopy equivalence. In point of fact, it was this somewhat roundabout line of thought that suggested (1.1).

We now take up the proof of (1.3). Consider the homomorphisms

$$
\begin{aligned}
& q_{*}: \pi_{j}\left(S^{25}\right) \longrightarrow \pi_{j}(\Sigma(W)) \\
& \quad \partial: \pi_{j+1}(E I V, \Sigma(W)) \longrightarrow \pi_{j}(\Sigma(W)) .
\end{aligned}
$$

Lemma 2.5. For $j \leqq 32$ there is a natural bijection $h: \pi_{j}\left(S^{25}\right) \rightarrow$ $\pi_{j+1}(E I V, \Sigma(W))$ such that $\partial \circ h=q_{*}$.

Proof. $q$ defines a map $\bar{q}:\left(e_{26}, S^{25}\right) \rightarrow(E I V, \Sigma(W))$ and by [11, Chapter XI, Ex. B-3] (cf. the references given there to [10] and [16]), $\bar{q}_{*}$ is bijective in dimensions $\leqq 33$. Let

$$
\gamma: \pi_{j}\left(S^{25}\right) \longrightarrow \pi_{j+1}\left(e_{26}, S^{25}\right), j \leqq 32,
$$

be the inverse of the boundary map. Then $h=\bar{q}_{*} \circ \gamma$ is as desired.

The first assertion of (1.3) follows immediately from (2.5). For the exactness of

$$
0 \longrightarrow \pi_{25}\left(S^{25}\right) \underset{q_{*}}{\longrightarrow} \pi_{25}(\Sigma(W)) \underset{E_{*}}{\longrightarrow} \pi_{25}(E I V) \longrightarrow 0
$$

we need only the following.

Lemma 2.6. $\partial: \pi_{26}(E I V, \Sigma(W)) \rightarrow \pi_{25}(\Sigma(W))$ is one-to-one.

Proof. From [8], $\pi_{j}\left(E I V, S^{9}\right) \approx \pi_{j-1}\left(S^{16}\right), j \leqq 31$. Thus, since $\pi_{26}\left(S^{9}\right)$ and $\pi_{25}\left(S^{16}\right)$ are finite groups, so is $\pi_{26}(E I V)$. Since $\pi_{26}(E I V, \Sigma(W)) \approx \boldsymbol{Z}$ by $(2.5)$, the map $\pi_{26}(E I V) \rightarrow \pi_{26}(E I V, \Sigma(W))$ is zero. The lemma follows by exactness.

The fact that $\pi_{25}(E I V)$ is a finite 2-primary group also follows from the results in [8], so we are left with the task of proving that the above sequence splits. (If it splits at all, the splitting is canonical, since $\pi_{25}(E I V)$ will have to be identified with the torsion subgroup of $\pi_{25}(\Sigma(W))$.)

The imbedding $S^{9} \rightarrow E I V$ studied in [8] defines a generator of $\pi_{9}(E I V) \approx Z$, hence $E$ can be assumed to define a map

$$
i:\left(\Sigma(W), S^{9}\right) \rightarrow\left(E I V, S^{9}\right), i \mid S^{9}=1,
$$

where $S^{9} \subset \Sigma(W)$ is given by our standard cellular decomposition of 
$\Sigma(W)$. Using $\pi_{25}\left(E I V, S^{9}\right) \approx \pi_{24}\left(S^{16}\right) \approx\left(Z_{2}\right)^{2}$ [8], we obtain a commutative diagram

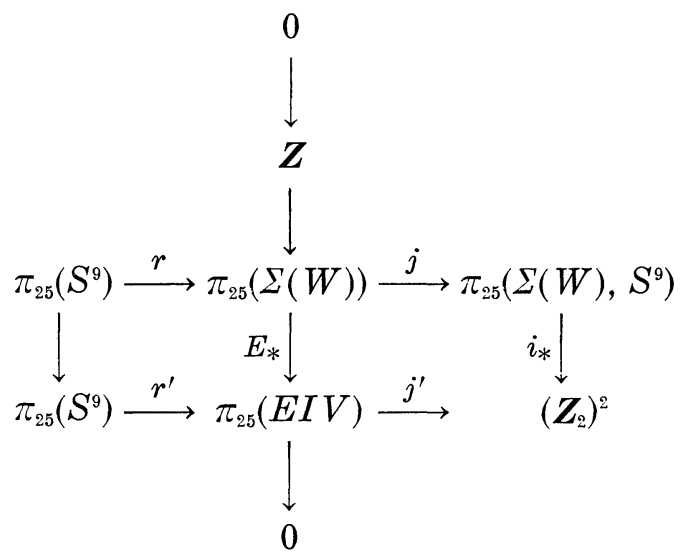

where the second column and both rows are exact. Extending this diagram two more terms to the right, one easily establishes the surjective half of the five lemma.

Lemma 2.7. $i_{*}: \pi_{25}\left(\Sigma(W), S^{9}\right) \rightarrow\left(\boldsymbol{Z}_{2}\right)^{2}$ is surjective and $\operatorname{Ker}\left(i_{*}\right) \subset \operatorname{Im}(j)$.

Lemma 2.8. $j^{-1}\left(\operatorname{Ker}\left(i_{*}\right)\right)=\operatorname{Ker}\left(E_{*}\right) \oplus \operatorname{Im}(r)$.

Proof. $j^{-1}\left(\operatorname{Ker}\left(i_{*}\right)\right)=\operatorname{Ker}\left(i_{*} \circ j\right)=\operatorname{Ker}\left(j^{\prime} \circ E_{*}\right) . \quad$ Now $\operatorname{Ker}\left(E_{*}\right)$ is infinite cyclic while $\operatorname{Im}(r)$ is a torsion group. Thus $\operatorname{Ker}\left(E_{*}\right) \cap \operatorname{Im}(r)=$ 0 . Furthermore, if $j^{\prime}\left(E_{*}(a)\right)=0$, then $E_{*}(a) \in \operatorname{Im}\left(r^{\prime}\right)$ and $a=b+c$, $b \in \operatorname{Ker}\left(E_{*}\right), c \in \operatorname{Im}(r)$.

Corollary 2.9. $\operatorname{Ker}\left(i_{*}\right)$ is the infinite cyclic group $j\left(\operatorname{Ker}\left(E_{*}\right)\right)$.

Lemma $2.10 . \quad\left(\boldsymbol{Z}_{2}\right)^{2} \subset \pi_{25}\left(\Sigma(W), S^{9}\right)$.

Proof. In $\Sigma(W)=S^{9} \bigcup_{g} e_{17}$, the attaching map $g$ defines the characteristic map

$$
\bar{g}:\left(e_{17}, S^{16}\right) \longrightarrow\left(\Sigma(W), S^{9}\right) .
$$

Since suspension $\Sigma: \pi_{24}\left(S^{16}\right) \rightarrow \pi_{25}\left(S^{17}\right)$ is one-to-one, it follows [11, p. 333] that

$$
\bar{g}_{*}: \pi_{25}\left(e_{17}, S^{16}\right) \longrightarrow \pi_{25}\left(\Sigma(W), S^{9}\right)
$$

is one-to-one. But $\pi_{25}\left(e_{17}, S^{16}\right) \approx \pi_{24}\left(S^{16}\right) \approx\left(Z_{2}\right)^{2}$. 
Proposition 2.11. $\operatorname{Ker}\left(E_{*}\right)$ is a direct summand of $\pi_{25}(\Sigma(W))$.

Proof. Write $\operatorname{Ker}\left(E_{*}\right) \subset \boldsymbol{Z}^{1}$, where $\boldsymbol{Z}^{1}$ stands for a maximal infinite cylic subgroup of $\pi_{25}(\Sigma(W))$. $\operatorname{Im}(r) \cap \boldsymbol{Z}^{1}=0$, so $j \mid \boldsymbol{Z}^{1}$ is one-to-one. Thus $j\left(\boldsymbol{Z}^{1}\right) \cap\left(\boldsymbol{Z}_{2}\right)^{2}=0$, and, by $(2.9)$, $\operatorname{Im}\left(i_{*}\right) \supset\left(\boldsymbol{Z}_{2}\right)^{2} \oplus j\left(\boldsymbol{Z}^{1}\right) / j\left(\operatorname{Ker}\left(E_{*}\right)\right)$. Thus $j\left(\operatorname{Ker}\left(E_{*}\right)\right)=j\left(\boldsymbol{Z}^{1}\right)$, so $\operatorname{Ker}\left(E_{*}\right)=\boldsymbol{Z}^{1}$.

This completes the proof of (1.3). It also proves

$$
\pi_{25}\left(\Sigma(W), S^{9}\right) \approx \boldsymbol{Z}+\left(\boldsymbol{Z}_{2}\right)^{2} .
$$

3. The homotopy sequence of $\left(\Sigma(W), S^{9}\right)$. For the computation of $\pi_{j}(E I V), j \leqq 24$, we are reduced to computing $\pi_{j}(\Sigma(W))$. We begin the attack on this latter problem by investigating the boundary operator $\partial$ in the homotopy sequence of $\left(\Sigma(W), S^{9}\right)$.

Recall that $\Sigma(W)=S^{9} \cup_{g} e_{17}$ where $g$ is the suspension of the standard Hopf map $f: S^{15} \rightarrow S^{8}$. By [11, p. 334] one shows that

$$
\bar{g}_{*}: \pi_{j}\left(e_{17}, S^{16}\right) \longrightarrow \pi_{j}\left(\Sigma(W), S^{9}\right)
$$

is bijective for $j \leqq 24, \bar{g}$ the characteristic map determined by $g$. Let

$$
F: \pi_{j}\left(\Sigma(W), S^{9}\right) \longrightarrow \pi_{j-1}\left(S^{16}\right), j \leqq 24,
$$

be the natural bijection obtained by composing $\left(\bar{g}_{*}\right)^{-1}$ with the natural isomorphism $\pi_{j}\left(e_{17}, S^{16}\right) \approx \pi_{j-1}\left(S^{16}\right)$.

Lemma 3.1. $\partial: \pi_{j}\left(\Sigma(W), S^{9}\right) \rightarrow \pi_{j-1}\left(S^{9}\right)$ is given by $g_{*} \circ F$ if $j \leqq 24$.

Next consider the commutative diagram $(n \leqq 29)$

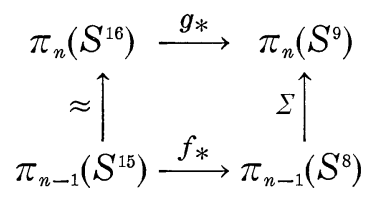

where the vertical maps are suspensions.

Lemma 3.2. $\operatorname{Ker}\left\{\partial: \pi_{j}\left(\Sigma(W), S^{9}\right) \rightarrow \pi_{\jmath-1}\left(S^{9}\right)\right\} \approx \operatorname{Im}\left(f_{*}\right) \cap \operatorname{Ker}(\Sigma)$ in $\pi_{j-2}\left(S^{8}\right), j \leqq 24$.

Proof. By (3.1) we are reduced to finding $\operatorname{Ker}\left(g_{*}\right)$. In the above diagram $f_{*}$ is injective (because it has Hopf invariant one [7, exposé 6, Proposition 5]). This immediately yields the assertion. 
We study $\operatorname{Im}\left(f_{*}\right) \cap \operatorname{Ker}(\Sigma)$ by means of the exact suspension sequence [7, expose 6]:

$$
\cdots \underset{\Sigma}{\longrightarrow} \pi_{n+1}\left(S^{9}\right) \underset{H}{\longrightarrow} \pi_{n}\left(\Omega\left(S^{9}\right), S^{8}\right) \underset{\Delta}{\longrightarrow} \pi_{n-1}\left(S^{8}\right) \underset{\Sigma}{\longrightarrow} \pi_{n}\left(S^{9}\right) \underset{H}{\longrightarrow} \cdots .
$$

This gives $\operatorname{Ker}(\Sigma)=\operatorname{Im}(\Delta)$. In order to study $\Delta$ we will consider the topology of $\Omega\left(S^{9}\right)$ in lower dimensions.

Let $i_{8}$ generate $\pi_{8}\left(S^{8}\right)$ and consider the Whitehead product $\left[i_{8}, i_{8}\right] \in \pi_{15}\left(S^{8}\right)$. Let $h: S^{15} \rightarrow S^{8}$ be in this homotopy class and set $X=$ $S^{8} \bigcup_{k} e_{16}$. It is known [7, exposé 5] that $\Omega\left(S^{9}\right)$ has the homotopy type of a $C W$ complex obtained by attaching to $X$ cells of dimensions $\geqq 24$. Thus the inclusion $\left(X, S^{8}\right) \subset\left(\Omega\left(S^{9}\right), S^{8}\right)$ is a homotopy equivalence in dimensions $\leqq 22$, and in this range we can consider $\Delta$ as defined on $\pi_{n}\left(X, S^{8}\right) . h$ determines a characteristic map

$$
\bar{h}:\left(e_{16}, S^{15}\right) \longrightarrow\left(X, S^{8}\right) \text {. }
$$

By [11, p. 334] we obtain

Lemma 3.3. $\bar{h}_{*}: \pi_{n}\left(e_{16}, S^{15}\right) \rightarrow \pi_{n}\left(X, S^{8}\right)$ is bijective, $n \leqq 22$.

COROLLARY 3.4. $\Delta=h_{*} \circ \partial \circ \bar{h}_{*}^{-1}$ in $\operatorname{dim} \leqq 22$, where

$$
\partial: \pi_{n}\left(e_{16}, S^{15}\right) \approx \pi_{n-1}\left(S^{15}\right) .
$$

COROLlaRY 3.5. $\operatorname{Ker}\left\{\partial: \pi_{j}\left(\Sigma(W), S^{9}\right) \rightarrow \pi_{j-1}\left(S^{9}\right)\right\} \approx \operatorname{Im}\left(f_{*}\right) \cap \operatorname{Im}\left(h_{*}\right)$ in $\pi_{j \sim 2}\left(S^{8}\right), j \leqq 23$.

4. $\pi_{j}(\Sigma(W)), j \leqq 18$. For the simple proof of the following lemma I am indebted to S. Araki.

Lemma 4.1. Let $g$ be the suspension of the standard Hopf map $f: S^{15} \rightarrow S^{8}$. The class $[g]$ generates $\pi_{16}\left(S^{9}\right) \approx \boldsymbol{Z}_{240}$.

Proof. Let $\sigma \in \pi_{7}(S O(8))$ be the element defined by the natural action on $R^{8}$ of the unit sphere of Cayley numbers. Let $\sigma^{\prime} \in \pi_{7}(S O(9))$ be the image of $\sigma$ under the standard inclusion $S O(8) \subset S O(9)$. Then $\sigma^{\prime}$ generates $\pi_{7}(S O(9)) \approx \boldsymbol{Z}[\mathbf{1 5}]$. The $J$-homomorphism

$$
J: \pi_{7}(S O(9)) \longrightarrow \pi_{16}\left(S^{9}\right) \approx \boldsymbol{Z}_{240}
$$

is surjective [12] and $J\left(\sigma^{\prime}\right)=[g]$.

COROLlary 4.2. $\pi_{16}(\Sigma(W))=0$

This establishes (1.4). For (1.5) and (1.6) we will need to make 
use of (3.5).

For $h$ and $f$ as in $\S 3$, the class $[\zeta]=[h]-2[f]$ is a torsion element in $\pi_{15}\left(S^{8}\right)$, hence $\zeta: S^{15} \rightarrow S^{8}$ is the suspension of some map [7, exposé 6].

Lemma 4.3. Let $\beta \in \pi_{16}\left(S^{15}\right) \approx \boldsymbol{Z}_{2}$ be the generator. Then $h_{*}(\beta)$ is a suspension class.

Proof. Since $\beta$ is a suspension class, $h_{*}(\beta)=2 f_{*}(\beta)+\zeta_{*}(\beta)=$ $\zeta_{*}(\beta)$ and this is a suspension class.

Corollary 4.4. $\operatorname{Ker}\left\{\partial: \pi_{18}\left(\Sigma(W), S^{9}\right) \rightarrow \pi_{17}\left(S^{9}\right)\right\}=0$.

Proof. By (4.3), $\operatorname{Im}\left(h_{*}\right)$ in $\pi_{10}\left(S^{8}\right)$ is contained in the image of the suspension. Therefore $\operatorname{Im}\left(f_{*}\right) \cap \operatorname{Im}\left(h_{*}\right)=0$ in $\pi_{16}\left(S^{8}\right)$. The conclusion follows by (3.5).

COROLlaRY 4.5. $\pi_{17}(\Sigma(W)) \approx \boldsymbol{Z}+\left(\boldsymbol{Z}_{2}\right)^{2}$.

Proof. $\pi_{18}\left(\Sigma(W), S^{9}\right) \approx \pi_{17}\left(S^{16}\right) \approx \boldsymbol{Z}_{2}$ by $(3.0)$, and $\pi_{17}\left(S^{9}\right) \approx\left(\boldsymbol{Z}_{2}\right)^{3}$. From the exact sequence of $\left(\Sigma(W), S^{9}\right)$ and (4.4) one obtains

$$
0 \longrightarrow\left(\boldsymbol{Z}_{2}\right)^{2} \longrightarrow \pi_{17}(\Sigma(W)) \longrightarrow \pi_{17}\left(\Sigma(W), S^{9}\right) \longrightarrow \pi_{16}\left(S^{9}\right) .
$$

Since $\pi_{17}\left(\Sigma(W), S^{9}\right) \approx Z$ and $\pi_{16}\left(S^{9}\right)$ is finite, this gives an exact sequence

$$
0 \longrightarrow\left(\boldsymbol{Z}_{2}\right)^{2} \longrightarrow \pi_{17}(\Sigma(W)) \longrightarrow \boldsymbol{Z} \longrightarrow 0 \text {. }
$$

This completes the proof of (1.5).

Proceeding analogously as above, let $\beta \in \pi_{17}\left(S^{15}\right) \approx \boldsymbol{Z}_{2}$ be the generator and show that $h_{*}(\beta) \in \operatorname{Im}(\Sigma)$. Then

$$
\partial: \pi_{19}\left(\Sigma(W), S^{9}\right) \longrightarrow \pi_{18}\left(S^{9}\right)
$$

is one-to-one. Since, by $(3.0), \pi_{19}\left(\Sigma(W), S^{9}\right) \approx \boldsymbol{Z}_{2}$, and $\pi_{18}\left(S^{9}\right) \approx\left(\boldsymbol{Z}_{2}\right)^{4}$, one obtains

$$
0 \longrightarrow\left(\boldsymbol{Z}_{2}\right)^{3} \longrightarrow \pi_{18}(\Sigma(W)) \longrightarrow \pi_{18}\left(\Sigma(W), S^{9}\right) \stackrel{\partial}{\longrightarrow} \cdots
$$

where $\partial$ is one-to-one by (4.4). This yields the following proposition and so proves $(1.6)$.

Proposition 4.6. $\pi_{18}(\Sigma(W)) \approx\left(\boldsymbol{Z}_{2}\right)^{3}$.

5. Partial determinations of $\pi_{j}(\Sigma(W)), j=19,20$. The 3-primary components of these two groups present a special problem. The 
ambiguities left by the partial determinations in this section will be removed in $\S 7$ by cohomological methods.

Lemma 5.1. $\Delta: \pi_{17}\left(X, S^{8}\right) \rightarrow \pi_{16}\left(S^{8}\right)$ is one-to-one.

Proof. Consider the exact sequence

$$
\pi_{17}\left(X, S^{8}\right) \stackrel{\Delta}{\longrightarrow} \pi_{16}\left(S^{8}\right) \stackrel{\Sigma}{\longrightarrow} \pi_{17}\left(S^{9}\right) \stackrel{H}{\longrightarrow} \cdots .
$$

$H$ is zero since $\pi_{17}\left(S^{9}\right)$ is finite. Thus $\Sigma$ is onto. Also $\pi_{16}\left(S^{8}\right) \approx\left(\boldsymbol{Z}_{2}\right)^{4}$, $\pi_{17}\left(S^{9}\right) \approx\left(\boldsymbol{Z}_{2}\right)^{3}$, so, by (3.3), $\operatorname{Im}(\Delta) \approx \boldsymbol{Z}_{2} \approx \pi_{17}\left(X, S^{8}\right)$. It follows that $\Delta$ is one-to-one.

Corollary 5.2. $\Delta: \pi_{18}\left(X, S^{8}\right) \rightarrow \pi_{17}\left(S^{8}\right)$ is one-to-one.

Proof. By (5.1) the sequence

$$
\pi_{18}\left(X, S^{8}\right) \stackrel{\Delta}{\longrightarrow} \pi_{17}\left(S^{8}\right) \stackrel{\Sigma}{\longrightarrow} \pi_{18}\left(S^{9}\right) \longrightarrow 0
$$

is exact. Since $\pi_{17}\left(S^{8}\right) \approx\left(\boldsymbol{Z}_{2}\right)^{5}, \pi_{18}\left(S^{9}\right) \approx\left(\boldsymbol{Z}_{2}\right)^{4}$, we obtain $\operatorname{Im}(\Delta)=$ $\operatorname{Ker}(\Sigma) \approx \boldsymbol{Z}_{2} \approx \pi_{18}\left(X, S^{8}\right)$.

Corollary 5.3. $\Delta: \pi_{19}\left(X, S^{8}\right) \rightarrow \pi_{18}\left(S^{8}\right)$ is one-to-one.

Proof. By (5.2)

$$
\pi_{19}\left(X, S^{8}\right) \stackrel{\Delta}{\longrightarrow} \pi_{18}\left(S^{8}\right) \stackrel{\Sigma}{\longrightarrow} \pi_{19}\left(S^{9}\right) \longrightarrow 0
$$

is exact.

$\pi_{18}\left(S^{8}\right) \approx\left(\boldsymbol{Z}_{24}\right)^{2}+\boldsymbol{Z}_{2}, \pi_{19}\left(S^{9}\right) \approx \boldsymbol{Z}_{24}+\boldsymbol{Z}_{2}$, and $\pi_{19}\left(X, S^{8}\right) \approx \pi_{18}\left(S^{15}\right) \approx \boldsymbol{Z}_{24}$. The assertion follows.

By (5.3) and (3.4), $h_{*}: \pi_{18}\left(S^{15}\right) \rightarrow \pi_{18}\left(S^{8}\right)$ is one-to-one. Let $\beta$ generate $\pi_{18}\left(S^{15}\right) \approx \boldsymbol{Z}_{24}$. Then $\beta$ is a suspension class and

$$
h_{*}(\beta)=2 f_{*}(\beta)+\zeta_{*}(\beta)
$$

is of order 24. Since $f_{*}$ is known to be one-to-one in all dimensions, $f_{*}(\beta)$ is also of order 24 . It follows that $\zeta_{*}(\beta)$ is of order 24 or 8 . This ambiguity affects the rest of this section.

LEMMA 5.4. $\partial: \pi_{20}\left(\Sigma(W), S^{9}\right) \rightarrow \pi_{19}\left(S^{9}\right)$ has kernel 0 or $\boldsymbol{Z}_{3}$.

Proof. If $\zeta_{*}(\beta)$ is order 24, then $\operatorname{Im}\left(f_{*}\right) \cap \operatorname{Im}\left(h_{*}\right)$ is 0 in $\pi_{18}\left(S^{8}\right)$. If $\zeta_{*}(\beta)$ is of order 8 , then $\operatorname{Im}\left(f_{*}\right) \cap \operatorname{Im}\left(h_{*}\right) \approx \boldsymbol{Z}_{3}$ in $\pi_{18}\left(S^{8}\right)$. The lemma follows by $(3.5)$. 
PRoposition 5.5. $\pi_{19}(\Sigma(W)) \approx Z_{2}$ or $Z_{6}$.

Proof. Consider the exact sequence

$0 \longrightarrow \operatorname{Ker}(\partial) \longrightarrow \pi_{20}\left(\Sigma(W), S^{9}\right) \stackrel{\partial}{\longrightarrow} \pi_{19}\left(S^{9}\right) \longrightarrow \pi_{19}(\Sigma(W)) \longrightarrow 0$

where exactness holds on the right by the proof of $(4.6)$.

$$
\pi_{20}\left(\Sigma(W), S^{9}\right) \approx \pi_{19}\left(S^{16}\right) \approx \boldsymbol{Z}_{24} \text { and } \pi_{19}\left(S^{9}\right) \approx \boldsymbol{Z}_{24}+\boldsymbol{Z}_{2} .
$$

The proposition follows by (5.4).

Proposition 5.6. There is an exact sequence

$$
0 \longrightarrow \boldsymbol{Z}_{504}+\boldsymbol{Z}_{2} \longrightarrow \pi_{20}(\Sigma(W)) \longrightarrow \pi_{19}(\Sigma(W)) \otimes \boldsymbol{Z}_{3} \longrightarrow 0 \text {. }
$$

Proof. By (5.4) and (5.5) the kernel of $\partial: \pi_{20}\left(\Sigma(W), S^{9}\right) \rightarrow \pi_{19}\left(S^{9}\right)$ is $\pi_{19}(\Sigma(W)) \otimes Z_{3}$. This, together with $\pi_{21}\left(\Sigma(W), S^{9}\right) \approx \pi_{20}\left(S^{16}\right) \approx 0$ and $\pi_{20}\left(S^{9}\right) \approx \boldsymbol{Z}_{504}+\boldsymbol{Z}_{2}$, yields the proposition.

6. $\pi_{j}(\Sigma(W)), 21 \leqq j \leqq 23$. One has $\pi_{21}\left(S^{9}\right) \approx 0$ and $\pi_{21}\left(\Sigma(W), S^{9}\right) \approx$ $\pi_{20}\left(S^{16}\right) \approx 0$, so the exact homotopy sequence of the pair yields the following proposition, completing the proof of (1.9).

Proposition 6.1. $\pi_{21}(\Sigma(W)) \approx 0$.

Now let $\beta$ generate $\pi_{21}\left(S^{15}\right) \approx \boldsymbol{Z}_{2}$. As usual, $h_{*}(\beta)=\zeta_{*}(\beta)$ so that $\operatorname{Im}\left(f_{*}\right) \cap \operatorname{Im}\left(h_{*}\right)$ is 0 in $\pi_{21}\left(S^{8}\right)$. Thus $\partial: \pi_{23}\left(\Sigma(W), S^{9}\right) \rightarrow \pi_{22}\left(S^{9}\right)$ is oneto-one.

Proposition 6.2. $\pi_{22}(\Sigma(W)) \approx \boldsymbol{Z}_{3}$.

Proof. $\pi_{23}\left(\Sigma(W), S^{9}\right) \approx \pi_{22}\left(S^{16}\right) \approx Z_{2}, \pi_{22}\left(S^{9}\right) \approx Z_{6}$, and

$$
\pi_{22}\left(\Sigma(W), S^{9}\right) \approx \pi_{21}\left(S^{16}\right) \approx 0 .
$$

By the above remarks we obtain an exact sequence

$$
0 \longrightarrow \boldsymbol{Z}_{2} \longrightarrow \boldsymbol{Z}_{6} \longrightarrow \pi_{22}(\Sigma(W)) \longrightarrow 0 \text {. }
$$

This also establishes (1.10). In order to prove (1.11) a slight change in approach is needed. The difficulty is that we are now out of the range of validity of (3.5).

There is an exact sequence

$$
\pi_{24}\left(\Sigma(W), S^{9}\right) \stackrel{\partial}{\longrightarrow} \pi_{23}\left(S^{9}\right) \longrightarrow \pi_{23}(\Sigma(W)) \longrightarrow 0
$$


where exactness on the right follows from the fact that $\partial$ is one-toone on $\pi_{23}\left(\Sigma(W), S^{9}\right)$. Substituting the known values of the first two groups (note that we are still in the range of validity for $(3.0)$ ) we obtain

$$
\boldsymbol{Z}_{5}+\boldsymbol{Z}_{3}+\boldsymbol{Z}_{16} \stackrel{\partial}{\longrightarrow} \boldsymbol{Z}_{16}+\boldsymbol{Z}_{4} \longrightarrow \pi_{23}(\Sigma(W)) \longrightarrow 0 .
$$

Our problem will be to compute $\operatorname{Ker}(\partial)$ in (6.3a).

Lemma 6.4. $\Delta: \pi_{22}\left(X, S^{8}\right) \rightarrow \pi_{21}\left(S^{8}\right)$ is one-to-one.

Proof. By (3.3), $\pi_{22}\left(X, S^{8}\right) \approx \pi_{21}\left(S^{15}\right) \approx \boldsymbol{Z}_{2}$, and $\pi_{21}\left(S^{8}\right) \approx \boldsymbol{Z}_{6}+\boldsymbol{Z}_{2}$, $\pi_{22}\left(S^{9}\right) \approx Z_{6}$. The suspension sequence of $\S 3$ then yields

$$
\boldsymbol{Z}_{2} \stackrel{\Delta}{\longrightarrow} \boldsymbol{Z}_{6}+\boldsymbol{Z}_{2} \stackrel{\Sigma}{\longrightarrow} \boldsymbol{Z}_{6}
$$

which necessitates $\Delta \neq 0$.

Corollary 6.5. $\quad \Sigma: \pi_{22}\left(S^{8}\right) \rightarrow \pi_{23}\left(S^{9}\right)$ is onto.

Recall that $f_{*}: \pi_{22}\left(S^{15}\right) \rightarrow \pi_{22}\left(S^{8}\right)$ and $\Sigma: \pi_{21}\left(S^{7}\right) \rightarrow \pi_{22}\left(S^{8}\right)$ are one-to-one and

$$
\pi_{22}\left(S^{8}\right)=\operatorname{Im}\left(f_{*}\right) \oplus \operatorname{Im}(\Sigma)
$$

Furthermore,

$$
\begin{aligned}
\operatorname{Im}\left(f_{*}\right) & \approx \boldsymbol{Z}_{5}+\boldsymbol{Z}_{3}+\boldsymbol{Z}_{16} \\
\operatorname{Im}(\Sigma) & \approx \boldsymbol{Z}_{3}+\boldsymbol{Z}_{8}+\boldsymbol{Z}_{4} \\
\pi_{23}\left(S^{9}\right) & \approx \boldsymbol{Z}_{16}+\boldsymbol{Z}_{4}
\end{aligned}
$$

It now follows from (6.5) that $\Sigma: \pi_{22}\left(S^{8}\right) \rightarrow \pi_{23}\left(S^{9}\right)$ must vanish on $\boldsymbol{Z}_{5}+\boldsymbol{Z}_{3} \subset \operatorname{Im}\left(f_{*}\right)$ but must be one-to-one on $\boldsymbol{Z}_{16} \subset \operatorname{Im}\left(f_{*}\right)$. The following lemma now holds by (3.2).

Lemma 6.6. $\operatorname{Ker}(\partial)$ in (6.3a) is $\boldsymbol{Z}_{5}+\boldsymbol{Z}_{3}$.

Proposition 6.7. $\pi_{23}(\Sigma(W)) \approx \boldsymbol{Z}_{4}$.

Proof. By (6.6), $\operatorname{Im}(\partial) \approx \boldsymbol{Z}_{16}$ in $(6.3 \mathrm{a})$. Regardless of how the imbedding $\operatorname{Im}(\partial) \subset \boldsymbol{Z}_{16}+\boldsymbol{Z}_{4}$ is realized, the quotient must be $\boldsymbol{Z}_{4}$.

This completes the proof of (1.11).

7. The 3-primary components in $\pi_{j}(E I V), j=19,20$. Our present aim is to complete the proofs of (1.7) and (1.8) which were begun in 
$\S 5$. Let $\Omega$ denote the space of loops on EIV. From the spectral sequence one easily obtains:

Lemma 7.1. In dimensions $<32, H^{*}\left(\Omega ; \boldsymbol{Z}_{3}\right)$ has a basis $\left\{1, x_{8}, x_{16}\right.$, $\left.x_{8}^{2}, x_{8} x_{16}, x_{24}\right\}, \operatorname{dim}\left(x_{i}\right)=i$. Furthermore, $x_{8}^{3}=0$.

In order to compute the 3-primary components of $\pi_{18}(\Omega)$ and $\pi_{19}(\Omega)$, we proceed by the method of killing cohomology classes in $H^{*}\left(\Omega ; \boldsymbol{Z}_{3}\right)$ via successive fibrations with appropriate Eilenberg-MacLane complexes as fibers. This yields the values of $\pi_{j}(\Omega) \otimes \boldsymbol{Z}_{3}, j=18,19$, and this information, together with $\S 5$, will prove (1.7) and (1.8). In the computations of this section we will also set the stage for computation of $\pi_{23}(\Omega) \otimes \boldsymbol{Z}_{3}$ which will be completed in $\S 8$.

A description the of $\boldsymbol{Z}_{3}$-algebra $H^{*}\left(\pi, n ; \boldsymbol{Z}_{3}\right), \pi$ a finitely generated abelian group, will be essential. Since, in $\S 8$, we will also need a description of $H^{*}\left(\pi, n ; \boldsymbol{Z}_{5}\right)$, we here discuss the general case of $H^{*}\left(\pi, n ; \boldsymbol{Z}_{p}\right), p$ an odd prime. For the proofs of our assertions cf. [6], especially exposés 9,15 , and 16 .

Let $I=\left(a_{1}, a_{2}, \cdots\right)$, a sequence of integers almost everywhere zero. $I$ will be called admissible if

$$
\begin{aligned}
& a_{i} \equiv 0 \text { or } 1 \bmod (2 p-2) \\
& a_{i} \geqq p a_{i+1} .
\end{aligned}
$$

The degree of $I$ is defined as $q(I)=\Sigma a_{i}$. $\quad I$ is said to be of the first kind if $a_{i} \neq 1, \forall i$. Otherwise $I$ is said to be of the second kind. If $I=\left(a_{1}, \cdots, a_{r}, 0,0, \cdots\right)$ is of the first kind, then one obtains an $I^{\prime}$ of the second kind by setting

$$
I^{\prime}=\left(a, \cdots, a_{r}, 1,0, \cdots\right) .
$$

Define the numbers

$$
\begin{aligned}
& g(I)=\left[p a_{1} /(p-1)\right]-q(I) \\
& n(I)=\left\{p a_{1} /(p-1)\right\}-q(I)
\end{aligned}
$$

where $[b]$ denotes the greatest integer $\leqq b$ and $\{b\}$ denotes the least integer $\geqq b$. Finally, let $P^{i}, i=0,1,2, \cdots$, denote the Steenrod reduced $p$-powers, $\beta$ the mod $p$ Bockstein, and define cohomology operations

$$
\begin{aligned}
& S t^{a}=P^{k}, b=2 k(p-1) \\
& S t^{b}=\beta P^{k}, b=2 k(p-1)+1 \\
& S t^{I}=S t^{\left(a_{1}\right)} \circ S t^{\left(a_{2}\right)} \circ \cdots, I \text { admissible. }
\end{aligned}
$$

Theorem 7.2. (H. Cartan) If I is admissible of the first kind and if $n\left(I^{\prime}\right) \leqq n$, then 


$$
S t^{I}: H^{n+1}\left(\pi, n ; \boldsymbol{Z}_{p}\right) \longrightarrow H^{n+q\left(I^{\prime}\right)}\left(\pi, n ; \boldsymbol{Z}_{p}\right)
$$

is a monomorphism. If also $n(I) \leqq n$, then

$$
S t^{I}: H^{n}\left(\pi, n ; \boldsymbol{Z}_{p}\right) \longrightarrow H^{n+q(I)}\left(\pi, n ; \boldsymbol{Z}_{p}\right)
$$

is a monomorphism. Let $A^{*}\left(\pi, n ; \boldsymbol{Z}_{p}\right)$ be the direct sum of the images of all of the above monomorphisms, graded by $n+q\left(I^{\prime}\right)$ and $n+q(I)$ respectively. Then the operations $S t^{I}$ define a graded homomorphism

$$
A^{*}\left(\pi, n ; \boldsymbol{Z}_{p}\right) \longrightarrow H^{*}\left(\pi, n ; \boldsymbol{Z}_{p}\right)
$$

which is an isomorphism onto the image of suspension

$$
\sigma: H^{*}\left(\pi, n+1 ; \boldsymbol{Z}_{p}\right) \longrightarrow H^{*}\left(\pi, n ; \boldsymbol{Z}_{p}\right) .
$$

Let $M_{n} \subset A^{*}\left(\pi, n ; \boldsymbol{Z}_{p}\right)$ be the graded subspace consisting of the direct sum of the images of those of the above monomorphisms where $I^{\prime}$ (respectively $\left.I\right)$ is required to satisfy the additional condition $g\left(I^{\prime}\right)<n$ (respectively $\left.g(I)<n\right)$. Then the algebra. $H^{*}\left(\pi, n ; \boldsymbol{Z}_{p}\right)$ is the free graded commutative $\boldsymbol{Z}_{p}$-algebra generated by $M_{n}$.

A further remark that is of use is that

$$
\begin{aligned}
H^{n}\left(\pi, n ; \boldsymbol{Z}_{p}\right) & \approx \operatorname{Hom}\left(\pi, \boldsymbol{Z}_{p}\right) \\
H^{n+1}\left(\pi, n ; \boldsymbol{Z}_{p}\right) & \approx \operatorname{Hom}\left({ }_{p} \pi, \boldsymbol{Z}_{p}\right)
\end{aligned}
$$

where ${ }_{p} \pi \subset \pi$ is the subgroup of elements of order $p$. One also notes that if ${ }_{p} \pi=\pi$, then

$$
\beta: H^{n}\left(\pi, n ; \boldsymbol{Z}_{p}\right) \longrightarrow H^{n+1}\left(\pi, n ; \boldsymbol{Z}_{p}\right)
$$

is a bijection.

In the remainder of this section we understand $p$ to be 3 . By the Adem relations [13] one has $P^{2}=P^{1} P^{1} . P^{1}, P^{3}$, and $\beta$ are trivial on $H^{*}\left(\Omega ; \boldsymbol{Z}_{3}\right)$ since the nontrivial dimensions in this graded vector space are all of the form $8 k$. Consequently $P^{2}$ is also trivial on $H^{*}\left(\Omega ; \boldsymbol{Z}_{3}\right)$.

We kill the class $x_{8} \in H^{8}\left(\Omega ; \boldsymbol{Z}_{3}\right)$ by a fibration

$$
K(Z, 7) \longrightarrow X_{1} \longrightarrow \Omega \text {. }
$$

An application of (7.2) gives the following classes as a basis of $H^{*}\left(\boldsymbol{Z}, 7 ; \boldsymbol{Z}_{3}\right)$ in dimensions $\leqq 25$ (where $\operatorname{dim}(y)=7$ ): $1, y P^{1}(y), \beta P^{1}(y)$, $P^{2}(y), \beta P^{2}(y), P^{3}(y), \beta P^{3}(y), P^{3} P^{1}(y), \beta P^{3} P^{1}(y), y \cdot P^{1}(y), y \cdot \beta P^{1}(y), y \cdot P^{2}(y)$, $y \cdot \beta P^{2}(y), P^{1}(y) \cdot \beta P^{1}(y),\left(\beta P^{1}(y)\right)^{2}$. By straightforward computations using the spectral sequence of this fibration, one obtains 
LeMmA 7.3. In $\operatorname{dim} \leqq 25, H^{*}\left(X_{1} ; Z_{3}\right)$ has basis $\left\{1, u_{11}, \beta\left(u_{11}\right), P^{1}\left(u_{11}\right)\right.$, $\left.\beta P^{1}\left(u_{11}\right), x_{16}, u_{19}, \beta\left(u_{19}\right), P^{3}\left(u_{11}\right), u_{11} \cdot \beta\left(u_{11}\right), u_{23}, \beta P^{3}\left(u_{11}\right),\left(\beta\left(u_{11}\right)\right)^{2}, x_{24}\right\}$, where the dimension of an element is indicated by its subscript.

In (7.3) the classes $x_{16}, x_{24}$ are the pull-backs of the classes in the base $\Omega$ that were denoted by the same symbols. $u_{11}$ and $u_{19}$ restrict respectively to $P^{1}(y)$ and $P^{3}(y)$ in the fiber. $u_{23}$ corresponds to $y \cdot x_{8}^{2}$ in the $E^{2}$ term of the spectral sequence. Using these facts and the Adem relations [13] one verifies the following relations:

$$
\begin{aligned}
\beta P^{1} \beta\left(u_{11}\right) & =0 \\
P^{2}\left(u_{11}\right) & =0 \\
P^{2} \beta\left(u_{11}\right) & =-\beta\left(u_{19}\right) \\
\beta P^{2} \beta\left(u_{11}\right) & =0 \\
P^{3} \beta\left(u_{11}\right) & =\beta P^{3}\left(u_{11}\right) \\
\beta P^{3} \beta\left(u_{11}\right) & =0 .
\end{aligned}
$$

Next kill $u_{11}$ by a fibration

$$
K\left(Z_{3}, 10\right) \longrightarrow X_{2} \longrightarrow X_{1} \text {. }
$$

By (7.2), a basis for $H^{*}\left(\boldsymbol{Z}_{3}, 10 ; \boldsymbol{Z}_{3}\right)$ in dimensions $\leqq 24$ is given by the following classes $(\operatorname{dim}(y)=10): 1, y, \beta(y), P^{1}(y), \beta P^{1}(y), P^{1} \beta(y), \beta P^{1} \beta(y)$, $P^{2}(y), \beta P^{2}(y), P^{2} \beta(y), \beta P^{2} \beta(y), y^{2}, y \cdot \beta(y), P^{3}(y), \beta P^{3}(y), P^{3} \beta(y), \beta P^{3} \beta(y)$, $y \cdot P^{1}(y)$.

Lemma 7.4. Transgression

$$
t: H^{15}\left(Z_{3}, 10 ; Z_{3}\right) \longrightarrow H^{16}\left(X_{1} ; Z_{3}\right)
$$

is bijective.

Proof. Otherwise the first nonvanishing $H^{i}\left(X_{2} ; \boldsymbol{Z}_{3}\right)$ for $i>0$ occurs for $i=15$, and this would give $\pi_{15}(\Omega) \otimes \boldsymbol{Z}_{3} \approx \pi_{15}\left(X_{2}\right) \otimes \boldsymbol{Z}_{3} \neq 0$, contradicting (1.4).

Applying all of this information to the spectral sequence of the fiber space $X_{2}$ we obtain.

LEMMA 7.5. In $\operatorname{dim} \leqq 24, H^{*}\left(X_{2} ; Z_{3}\right)$ has a basis $\left\{1, u_{16}, u_{18}, \beta\left(u_{18}\right)\right.$, $\left.u_{19}, P^{1}\left(u_{16}\right), P^{1} \beta\left(u_{18}\right), u_{23}, P^{2}\left(u_{16}\right), x_{24}\right\}$.

These classes satisfy the following relations: 


$$
\begin{aligned}
P^{2}\left(u_{16}\right) & \equiv-\beta P^{1} \beta\left(u_{18}\right) \bmod x_{24} \\
\beta\left(x_{24}\right) & =0 \\
\beta P^{2}\left(u_{16}\right) & =0 \text { (a consequence of the above two) } \\
\beta\left(u_{19}\right) & =0 \\
P^{1}\left(u_{19}\right) & \equiv 0 \bmod u_{23} \\
\beta P^{1}\left(u_{19}\right) & \equiv 0 \bmod x_{24} .
\end{aligned}
$$

Note that, by $(1.5), \pi_{16}(\Omega) \approx \boldsymbol{Z}+\left(\boldsymbol{Z}_{2}\right)^{2}$, hence to kill $u_{16}$ we need a fibration

$$
K(Z, 15) \longrightarrow X_{3} \longrightarrow X_{2} \text {. }
$$

Using (7.2), (7.5), and the above relations, we obtain.

Lemma 7.6. In $\operatorname{dim} \leqq 24, H^{*}\left(X_{3} ; Z_{3}\right)$ has a basis $\left\{1, u_{18}, \beta\left(u_{18}\right)\right.$, $\left.u_{19}, u_{20}, P^{1} \beta\left(u_{18}\right), u_{23}, P^{1}\left(u_{20}\right), x_{24}\right\}$ satisfying the relations: $\beta P^{1} \beta\left(u_{18}\right) \equiv 0$ $\bmod x_{24} ; \beta\left(u_{19}\right)=0 ; P^{1}\left(u_{19}\right) \equiv 0 \bmod u_{23} ; \beta P^{1}\left(u_{19}\right) \equiv 0 \bmod x_{24}$.

Corollary 7.7. $\pi_{18}(\Omega) \approx \boldsymbol{Z}_{6}$.

Proof. By $(7.6), \pi_{18}(\Omega) \otimes \boldsymbol{Z}_{3} \approx \boldsymbol{Z}_{3}$. By $(5.5), \pi_{18}(\Omega) \approx \boldsymbol{Z}_{2}$ or $\boldsymbol{Z}_{6}$.

This completes the proof of (1.7).

Next we kill $u_{18}$ by

$$
K\left(Z_{3}, 17\right) \longrightarrow X_{4} \longrightarrow X_{3} \text {. }
$$

Using the spectral sequence and (7.6) one readily obtains:

LemMA 7.8. $H^{j}\left(X_{4} ; Z_{3}\right) \approx 0,0<j<19$, and $H^{19}\left(X_{4} ; Z_{3}\right) \approx Z_{3}$.

CoRollaRY $7.9 . \quad \pi_{19}(\Omega) \approx \boldsymbol{Z}_{1512}+\boldsymbol{Z}_{\varrho}$.

Proof. By (5.6) and (7.7) there is an exact sequence

$$
0 \longrightarrow \boldsymbol{Z}_{9}+\boldsymbol{Z}_{8}+\boldsymbol{Z}_{7}+\boldsymbol{Z}_{2} \longrightarrow \pi_{19}(\Omega) \longrightarrow \boldsymbol{Z}_{3} \longrightarrow 0 \text {. }
$$

By $(7.8), \pi_{19}(\Omega) \otimes \boldsymbol{Z}_{3} \approx \boldsymbol{Z}_{3}$. Hence $\pi_{19}(\Omega) \approx \boldsymbol{Z}_{27}+\boldsymbol{Z}_{8}+\boldsymbol{Z}_{7}+\boldsymbol{Z}_{2}$.

This completes the proof of (1.8). Evidently in the above lemmas we have obtained information on the cohomology of the spaces $X_{i}$ in dimensions higher than necessary for the purposes of this section. This information will be used in the next section to help prove (1.12).

8. Partial determination of $\pi_{24}(E I V)$. Notice that by the theory of [8] there is an exact sequence 


$$
\pi_{24}\left(S^{16}\right) \longrightarrow \pi_{24}\left(S^{9}\right) \longrightarrow \pi_{24}(E I V) \longrightarrow \pi_{23}\left(S^{16}\right) \longrightarrow \pi_{23}\left(S^{9}\right)
$$

which gives explicitly

$$
\left(\boldsymbol{Z}_{2}\right)^{2} \longrightarrow \boldsymbol{Z}_{240}+\left(\boldsymbol{Z}_{2}\right)^{3} \longrightarrow \pi_{24}(E I V) \longrightarrow \boldsymbol{Z}_{240} \longrightarrow \boldsymbol{Z}_{16}+\boldsymbol{Z}_{4} .
$$

Thus, to prove (1.12) we must compute $\pi_{24}(E I V) \otimes \boldsymbol{Z}_{5}$ and $\pi_{24}(E I V) \otimes \boldsymbol{Z}_{3}$.

Recall the fibration $K\left(\boldsymbol{Z}_{3}, 17\right) \rightarrow X_{4} \rightarrow X_{3}$. Recall also from (7.6) the relation $\beta P^{1} \beta\left(u_{18}\right) \equiv 0 \bmod x_{24}$. Replacing $x_{24}$ with its negative if necessary, we obtain just two possibilities:

$$
\beta P^{1} \beta\left(u_{18}\right)=0
$$

or

$$
\beta P^{1} \beta\left(u_{18}\right)=x_{24} .
$$

In order to determine a basis for $H^{*}\left(X_{4} ; \boldsymbol{Z}_{3}\right)$ it will be necessary to consider these two possibilities.

Lemma 8.2. If $\beta P^{1} \beta\left(u_{18}\right)=0$, then, in $\operatorname{dim} \leqq 24, H^{*}\left(X_{4} ; Z_{3}\right)$ has as a basis $\left\{1, u_{19}, u_{20}, u_{21}, \beta\left(u_{21}\right), u_{23}, P^{1}\left(u_{20}\right), w_{23}, x_{24}\right\}$. The following relations are also satisfied: $\beta\left(u_{19}\right)=0 ; P^{1}\left(u_{19}\right) \equiv 0 \bmod u_{23} ; \beta P^{1}\left(u_{19}\right) \equiv 0 \bmod x_{24}$.

LEMMA 8.3. If $\beta P^{1} \beta\left(u_{18}\right)=x_{24}$, then, in $\operatorname{dim} \leqq 24, H^{*}\left(X_{4} ; \boldsymbol{Z}_{3}\right)$ has as a basis $\left\{1, u_{19}, u_{20}, u_{21}, \beta\left(u_{21}\right), P^{1}\left(u_{20}\right), u_{23}\right\}$ with $\beta\left(u_{19}\right)=0, \beta P^{1}\left(u_{19}\right)=0$, $P^{1}\left(u_{19}\right) \equiv 0 \bmod u_{23}$.

We kill $u_{19}$ by

$$
K\left(\boldsymbol{Z}_{27}, 18\right) \longrightarrow X_{5} \longrightarrow X_{4} \text {. }
$$

The use of $K\left(\boldsymbol{Z}_{27}, 18\right)$ is dictated by (7.9). The 3-primary component of $\pi_{19}\left(X_{5}\right)$ is 0 .

Note that by $(7.2)$ a basis of $H^{*}\left(\boldsymbol{Z}_{27}, 18 ; \boldsymbol{Z}_{3}\right)$ is given by $\left\{1, y_{18}, y_{19}\right.$, $\left.P^{1}\left(y_{18}\right), \beta P^{1}\left(y_{18}\right), P^{1}\left(y_{19}\right), \beta P\left(y_{19}\right)\right\}$ in $\operatorname{dim} \leqq 24$. Here $\beta\left(y_{18}\right)=0$.

LEMMA 8.4. Transgression

$$
t: H^{19}\left(\boldsymbol{Z}_{27}, 18 ; \boldsymbol{Z}_{3}\right) \longrightarrow H^{20}\left(X_{4} ; Z_{3}\right)
$$

is bijective.

Proof. Otherwise, $\pi_{19}\left(X_{5}\right) \otimes \boldsymbol{Z}_{3} \approx \boldsymbol{Z}_{3}$, contradicting the construction of $X_{5}$.

Corollary 8.5. $H^{i}\left(X_{5}, \boldsymbol{Z}_{3}\right) \approx 0,0<i<21$, while $H^{21}\left(X_{5} ; \boldsymbol{Z}_{3}\right) \approx \boldsymbol{Z}_{3}$ and is generated by (the pull-back of) $u_{21} . \quad \beta\left(u_{21}\right) \neq 0$. 
Lemma 8.6. $t\left(P^{1}\left(y_{18}\right)\right)= \pm u_{23}$.

Proof. In either the hypothesis of (8.2) or of (8.3), $t\left(P^{1}\left(y_{18}\right)\right)=$ $P^{1}\left(u_{19}\right) \equiv 0 \bmod u_{23}$. We must show $P^{1}\left(u_{19}\right) \neq 0$. Suppose the contrary. Then, killing $u_{21}$ by $K\left(\boldsymbol{Z}_{3}, 20\right) \rightarrow X_{6} \rightarrow X_{5}$, one shows that $H^{i}\left(X_{6} ; \boldsymbol{Z}_{3}\right) \approx 0$, $0<i<22$, and $H^{22}\left(X_{6} ; \boldsymbol{Z}_{3}\right) \approx \boldsymbol{Z}_{3}$. Thus $\pi_{22}(\Omega) \otimes \boldsymbol{Z}_{3} \approx \pi_{22}\left(X_{6}\right) \otimes \boldsymbol{Z}_{3} \approx \boldsymbol{Z}_{3}$, contradicting (1.11).

Lemma 8.7. In the hypothesis of (8.2), $t\left(\beta P^{1}\left(y_{18}\right)\right)= \pm x_{24}$.

Proof. By (8.2), $t\left(\beta P^{1}\left(y_{18}\right)\right)=\beta P^{1}\left(u_{19}\right) \equiv 0 \bmod x_{24}$. We must show $\beta P^{1}\left(u_{19}\right) \neq 0$. Suppose the contrary. Kill $u_{21} \in H^{21}\left(X_{5} ; \boldsymbol{Z}_{3}\right)$ by $K\left(\boldsymbol{Z}_{3}, 20\right) \rightarrow$ $X_{6} \rightarrow X_{5}$. Using (8.2), (8.4), (8.5), and (8.6), one shows $\pi_{23}(\Omega) \otimes \boldsymbol{Z}_{3} \approx$ $\pi_{23}\left(X_{6}\right) \otimes \boldsymbol{Z}_{3} \approx \boldsymbol{Z}_{3}+\boldsymbol{Z}_{3}$. Here the two generators of $H^{23}\left(X_{6} ; \boldsymbol{Z}_{3}\right)$ come from the $w_{23}$ of (8.2) and from $\beta P^{1}\left(y_{18}\right)$. This information, together with (8.1), implies that the 3-component of $\pi_{23}(\Omega)$ is $\boldsymbol{Z}_{3}+\boldsymbol{Z}_{3}$. Thus if $w_{23}, v_{23} \in H^{23}\left(X_{6} ; \boldsymbol{Z}_{3}\right)$ are the two generators, $\beta\left(w_{23}\right)$ and $\beta\left(v_{23}\right)$ will be linearly independent. But $\beta\left(w_{23}\right)$ and $\beta\left(v_{23}\right)$ are $\equiv 0 \bmod x_{24}$, so that we have reached a contradiction.

LEMMA 8.8. In the hypothesis of (8.3), $t\left(\beta P^{1}\left(y_{18}\right)\right)=0$.

Proof. $t\left(\beta P^{1}\left(y_{18}\right)=\beta P^{1}\left(u_{19}\right)=0\right.$ by $(8.3)$.

Putting all of this information together, one obtains.

LEMma 8.9. In either the hypothesis of (8.2) or of (8.3), $H^{*}\left(X_{5}, \boldsymbol{Z}_{3}\right)$ has as a basis in dim $\leqq 23$ classes $1, u_{21}, \beta\left(u_{21}\right), w_{23}$.

Proposition 8.10. The 3-primary component of $\pi_{23}(\Omega)$ is $\boldsymbol{Z}_{9}$.

Proof. By (8.9) and the process of killing $u_{21}$, one finds $\pi_{23}(\Omega) \otimes$ $\boldsymbol{Z}_{3} \approx \boldsymbol{Z}_{3}$. The assertion now follows by $(8.1)$.

There remains the task of finding the 5-primary component of $\pi_{24}(E I V)$. Here we make use of (1.2) and of the mod 5 Steenrod algebra. Recall from $[3,19.6]$ that if $x_{i}$ generates $H^{i}\left(\Sigma(W) ; Z_{5}\right), i=9,17$, then $P^{1}\left(x_{9}\right)= \pm 2 x_{17}$.

Kill $x_{9}$ by

$$
K(\boldsymbol{Z}, 8) \longrightarrow X_{1} \longrightarrow \Sigma(W) \text {. }
$$

This gives the following lemma. 
LEMMA 8.11. In $\operatorname{dim} \leqq 25, H^{*}\left(X_{1} ; Z_{5}\right)$ has a basis $\left\{1, u_{17}, u_{24}\right.$, $\left.\beta\left(u_{24}\right), u_{25}\right\}$ with relations $\beta\left(u_{17}\right)=0, P^{1}\left(u_{17}\right) \equiv \beta\left(u_{24}\right) \bmod u_{25}$.

Since $\pi_{17}(\Sigma(W)) \approx \boldsymbol{Z}+\left(\boldsymbol{Z}_{2}\right)^{2}$, one needs

$$
K(Z, 16) \longrightarrow X_{2} \longrightarrow X_{1}
$$

to kill $u_{17}$.

LEMMA 8.12. $H^{i}\left(X_{2} ; \boldsymbol{Z}_{5}\right) \approx 0,0<i<24$, and $H^{24}\left(X_{2} ; \boldsymbol{Z}_{5}\right) \approx \boldsymbol{Z}_{5}$.

COROLlary 8.13. The 5-primary component of $\pi_{24}(\Sigma(W))$ is $\boldsymbol{Z}_{25}$.

Proof. By $(8.12), \pi_{24}(\Sigma(W)) \otimes \boldsymbol{Z}_{5} \approx \boldsymbol{Z}_{5}$. The corollary now follows by $(8.1)$.

Now by (8.1), (8.10), and (8.13) we can conclude (1.12).

\section{REFERENCES}

1. S. Araki, Cohomology modulo 2 of the compact exceptional groups $E_{6}$ and $E_{7}, \mathrm{~J}$. Math. Osaka City Univ. (1-2) 12 (1961), 43-65.

2. - On root systems and an infinitesimal classification of irreducible symmetric spaces, J. Math. Osaka City Univ. 13 (1962), 1-34.

3. A. Borel and F. Hirzebruch, Characteristic classes and homogeneous spaces, Part $I$, Amer. J. Math. 80 (1958), 459-538.

4. R. Bott, The stable homotopy of the classical groups, Ann. of Math. (2) 70 (1959), 313-337.

5. - The space of loops on a Lie group, Michigan Math. J. 5 (1958), 35-61.

6. H. Cartan (et al.), Algèbres d'Eilenberg-MacLane et Homotopie, Seminaire H. Cartan (1954/55), Paris, 1955. (Mimeographed notes)

7. - Invariant de Hopf et Opérations Cohomologiques Secondaires, Seminaire

H. Cartan (1958/59), Paris, 1959. (Mimeographed notes)

8. L. Conlon, On the topology of EIII and EIV, Proc. Amer. Math. Soc. 16 (1965), 575-581.

9. B. Harris, Suspensions and characteristic maps for symmetric spaces (2) 76 (1962), 295-305.

10. P. J. Hilton, Suspension theorems and the generalized Hopf invariant, Proc. London Math. Soc. (3) 1 (1959), 462-492.

11. S. Hu, Homotopy Theory, Academic Press, New York, 1959.

12. J. Milnor and M. Kervaire, Bernoulli numbers, homotopy groups, and a theorem of Rohlin, Proc. Int. Congress of Math., Edinburgh, 1958, 454-458.

13. N. E. Steenrod and D. B. A. Epstein, Cohomology Operations, Princeton University Press, Princeton, New Jersey, 1962.

14. H. Toda, Composition Methods in Homotopy Groups of Spheres, Princeton University Press, Princeton, New Jersey, 1962.

15. H. Toda, Y. Saito, and I. Yokota, Note on the generator of $\pi_{7}(S O(n))$, Mem. College of Sci., Univ. of Kyoto 30 (1957), 227-230.

16. J. H. C. Whitehead, Note on suspension, Quart. J. Math., Oxford (2) 1 (1950), 9-22.

Received August 31, 1965.

LOyola UNIVERSity of NEW ORLEANS 


\section{PACIFIC JOURNAL OF MATHEMATICS}

\section{EDITORS}

H. SAMELSON

Stanford University

Stanford, California

J. P. JANS

University of Washington

Seattle, Washington 98105

\section{J. DuGUNDJI}

University of Southern California

Los Angeles, California 90007

RICHARD ARENS

University of California

Los Angeles, California 90024

\section{ASSOCIATE EDITORS}

E. F. BECKENBACH
B. H. NEUMANN

F. WOLF

K. YoSIDA

\section{SUPPORTING INSTITUTIONS}

\author{
UNIVERSITY OF BRITISH COLUMBIA \\ CALIFORNIA INSTITUTE OF TECHNOLOGY \\ UNIVERSITY OF CALIFORNIA \\ MONTANA STATE UNIVERSITY \\ UNIVERSITY OF NEVADA \\ NEW MEXICO STATE UNIVERSITY \\ OREGON STATE UNIVERSITY \\ UNIVERSITY OF OREGON \\ OSAKA UNIVERSITY \\ UNIVERSITY OF SOUTHERN CALIFORNIA
}

\author{
STANFORD UNIVERSITY \\ UNIVERSITY OF TOKYO \\ UNIVERSITY OF UTAH \\ WASHINGTON STATE UNIVERSITY \\ UNIVERSITY OF WASHINGTON \\ AMERICAN MATHEMATICAL SOCIETY \\ CHEVRON RESEARCH CORPORATION \\ TRW SYSTEMS \\ NAVAL ORDNANCE TEST STATION
}

Mathematical papers intended for publication in the Pacific Journal of Mathematics should be typewritten (double spaced). The first paragraph or two must be capable of being used separately as a synopsis of the entire paper. It should not contain references to the bibliography. Manuscripts may be sent to any one of the four editors. All other communications to the editors should be addressed to the managing editor, Richard Arens at the University of California, Los Angeles, California 90024 .

50 reprints per author of each article are furnished free of charge; additional copies may be obtained at cost in multiples of 50 .

The Pacific Journal of Mathematics is published monthly. Effective with Volume 16 the price per volume (3 numbers) is $\$ 8.00$; single issues, $\$ 3.00$. Special price for current issues to individual faculty members of supporting institutions and to individual members of the American Mathematical Society: $\$ 4.00$ per volume; single issues $\$ 1.50$. Back numbers are available.

Subscriptions, orders for back numbers, and changes of address should be sent to Pacific Journal of Mathematics, 103 Highland Boulevard, Berkeley 8, California.

Printed at Kokusai Bunken Insatsusha (International Academic Printing Co., Ltd.), No. 6, 2-chome, Fujimi-cho, Chiyoda-ku, Tokyo, Japan.

\section{PUBLISHED BY PACIFIC JOURNAL OF MATHEMATICS, A NON-PROFIT CORPORATION}

The Supporting Institutions listed above contribute to the cost of publication of this Journal, but they are not owners or publishers and have no responsibility for its content or policies. 


\section{Pacific Journal of Mathematics

Vol. 19, No. $3 \quad$ July, 1966

S. J. Bernau, The spectral theorem for unbounded normal operators .......

Lu-san Chen, Asymptotic behavior of solutions of parabolic equations of

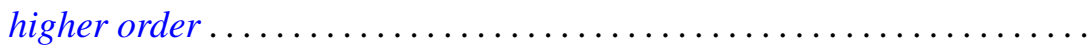

Lawrence William Conlon, An application of the Bott suspension map to the

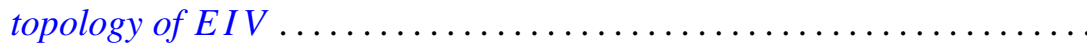

Neal Eugene Foland and John M. Marr, Sets with zero-dimensional kernels .........................................

Stanley Phillip Franklin and R. H. Sorgenfrey, Closed and image-closed

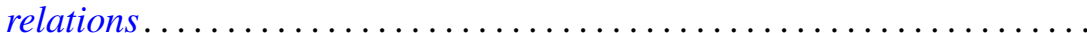

William Jesse Gray, A note on topological transformation groups with a

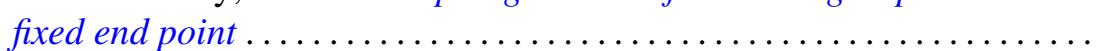

Myron Goldstein, $K$ - and L-kernels on an arbitrary Riemann surface ...... 449

George Joseph Kertz and Francis Regan, The exponential analogue of a generalized Weierstrass series .............................

Walter Leighton, On Liapunov functions with a single critical point ........ 467

Bernard Werner Levinger and Richard Steven Varga, On a problem of $O$.

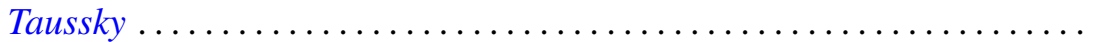

Lowell Duane Loveland, Tame subsets of spheres in $E^{3} \ldots \ldots \ldots \ldots \ldots . .489$

Erik Andrew Schreiner, Modular pairs in orthomodular lattices ......... 519

K. N. Srivastava, On dual series relations involving Laguerre polynomials ...............................

Arthur Steger, Diagonability of idempotent matrices.....

Walter Strauss, On continuity of functions with values in various Banach spaces...

Robert Vermes, On the zeros of a linear combination of polynomials ...

Elliot Carl Weinberg, On the scarcity of lattice-ordered matrix rings ....

Harold Widom, Toeplitz operators on $H_{p} \ldots \ldots \ldots \ldots \ldots$

Neal Zierler, On the lattice of closed subspaces of Hilbert space...

Irving Leonard Glicksberg, Correction to: "Maximal algebras and a theorem of Rado"

John Spurgeon Bradley, Correction to: "Adjoint quasi-differential operators of Euler type"

William Branham Jones, Erratum: "Duality and types of completeness in locally covex spaces".

Stanley P. Gudder, Erratum: "Uniqueness and existence properties of bounded observables" 